\title{
Familia transnacional y redefinición de los roles de género. El caso de la migración boliviana en España
}

\section{Sònia Parella Rubio}

Universidad Autónoma de Barcelona. Departamento de Sociología sonia.parella@uab.es

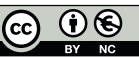

\section{Resumen}

El artículo analiza las prácticas y subjetividades de las familias bolivianas transnacionales en España. Se identifican los ajustes transnacionales en términos de renegociación de las responsabilidades de cuidado y de asignación de recursos, así como sus impactos en los roles de género. A través de un diseño metodológico cualitativo y bilocal, mediante el uso de la entrevista en profundidad a migrantes bolivianos y a los miembros adultos de la familia transnacional, el texto examina dos dimensiones: los cambios en las percepciones sobre los roles familiares de género en relación con el momento premigratorio y las transformaciones de las prácticas relacionadas con la división sexual del trabajo.

Palabras clave: migraciones; transnacionalismo; división sexual del trabajo; Bolivia.

\section{Abstract: Transnational family and the redefinition of gender roles: The case of Bolivian migrants in Spain}

This paper sheds light on the practices and subjectivities of Bolivian transnational families in Spain. We identify transnational family arrangements in terms of the renegotiation of caregiving responsibilities and the allocation of resources, as well as their impacts on gender roles. Drawing on a multi-site qualitative research design using in-depth interviews with Bolivian migrants and the adult family members left behind, the paper examines two dimensions: changing perceptions on family gender roles in comparison to the premigration stage and changes in practices resulting in a sexual division of labour.

Keywords: migrations; transnationalism; sexual division of labour; Bolivia.

\section{Sumario}

\section{Introducción}

2. El contexto migratorio boliviano hacia España: un nuevo esquema que transforma los patrones migratorios bolivianos «tradicionales»

3. Metodología de la investigación
4. Arreglos y formas de gestión de los vínculos transnacionales y sus impactos en los roles de género: el caso boliviano

5. Conclusiones

Referencias bibliográficas 


\section{Introducción ${ }^{1}$}

Este artículo analiza el vivir transnacional de las migrantes y los migrantes bolivianos residentes en España desde una perspectiva transnacional, con el fin de abordar los significados y las prácticas que delimitan los ajustes de las familias transformadas a partir de la migración internacional. Los procesos microsociales que generan los actuales flujos migratorios exigen estrategias a los miembros de la familia, que deben adaptarse a espacios geográficamente dispersos. Se trata de relaciones e intercambios de recursos, tangibles e intangibles, que implican tanto a los que se van como a los que permanecen (family arrangements) (Parreñas, 2001; Ho, 2002).

En concreto, se pretenden identificar los arreglos y las formas de gestión de los vínculos transnacionales y sus impactos en los roles de género. La hipótesis de partida es que tanto los cambios en las percepciones sobre los roles familiares de género, como los ajustes en la división sexual del trabajo en el interior de la familia transnacional, dependerán de si se trata de una emigración pionera masculina o femenina. Asimismo, dichos cambios y ajustes no admiten considerar el proceso migratorio como única variable explicativa generadora de efectos unívocos. Se asume que la migración en sí misma constituye un factor de cambio en las relaciones de género, cuya concreción dependerá de la estructura de oportunidades (factores económicos, sociales, históricos, etc.) de las personas en los contextos de origen y de destino (Magliano, 2007).

La perspectiva transnacional como marco para abordar las migraciones internacionales permite superar los modelos de análisis focalizados en el estado-nación receptor, que no dan cuenta de procesos a través de los cuales las migrantes y los migrantes construyen campos sociales que vinculan las zonas emisoras con las de destino (Basch et al., 1994). Esta perspectiva nos remite a la creciente intensidad de flujos poliédricos de personas, objetos, información y símbolos más allá de las fronteras nacionales. A través de las prácticas transnacionales, los migrantes construyen y reconstruyen sus vidas simultáneamente imbricadas en más de una sociedad a través de sus actividades cotidianas. De acuerdo con Basch et al. (1994: 238), «los procesos familiares y las relaciones entre personas definidas a través del parentesco, constituyen la base del resto de relaciones sociales transnacionales» ${ }^{2}$.

1. Los resultados presentados son parte del proyecto Transnacionalismo económico: remesas y empresas de los migrantes bolivianos en España (referencia SEJ2007-60734), financiado por el Ministerio de Investigación y Ciencia y dirigido por la catedrática Carlota Solé. El trabajo de campo de dicha investigación ha sido coordinado por Leonardo Cavalcanti (UAB) y por Leonardo de la Torre (Universidad San Simón, Bolivia). Asimismo, agradezco a Margarita Barañano y Fernando J. García (GRESCO, UCM) las discusiones y los debates en el transcurso de las jornadas internacionales "Vidas transmigrantes: hogares y género», que organizaron en la UCM los días 30 de noviembre y 1 de diciembre de 2011.

2. Aunque los vínculos transnacionales sean mucho más intensos en la actualidad, cabe preguntarse si todos los migrantes y sus descendientes desarrollan prácticas transnacionales, así como en qué medida muchos de los vínculos transnacionales, cuando se dan, tienen efectos en todas y cada una de las esferas de sus vidas. Las aportaciones de Alejandro Portes 
La familia transnacional permite superar las definiciones clásicas que conciben la familia como grupo de personas vinculadas por lazos de parentesco que configuran un hogar o lugar de residencia común (Ribas, 2001; Zontini, 2010). Del mismo modo que el hogar puede establecerse con o sin lazos de parentesco (hay hogares cuyos integrantes no tienen lazos familiares entre sí), es posible identificar familias en base a los lazos afectivos y a la construcción simbólica de unidad de las personas que forman parte de ella, aunque no compartan un mismo hogar (Puyana et al., 2009).

A la hora de delimitar el concepto de "familia transnacional», uno de los trabajos más aclamados es el de Bryceson y Vuorela (2002). Estas autoras la definen como «aquella familia cuyos miembros viven una parte o la mayor parte del tiempo separados los unos de los otros, siendo capaces de crear vínculos que permiten que sus miembros se sientan parte de una unidad y perciban su bienestar desde una dimensión colectiva, a pesar de la distancia física» (2002: 2). De esta definición, se desprende que el elemento definitorio de la estructura familiar transnacional no es la distancia, sino la existencia de un sentimiento de pertenencia por parte de sus miembros y que éstos participen colectivamente en la gestión del bienestar (Levitt y Glick Schiller, 2004).

Aunque tanto las personas que emigran como las que permanecen conforman nuevos hogares, es la existencia de vínculos generadores de sentimiento de unidad, así como la proyección de sus miembros hacia un futuro hogar imaginado compartido, lo que sostiene la estructura familiar transnacional. Tales vínculos pueden mantenerse, reforzarse o bien debilitarse, en la medida que los miembros modifiquen sus lealtades o inicien nuevas relaciones afectivas que puedan alterar las pertenencias a la familia transnacional que en su momento configuraron.

Dicha articulación transnacional, además, se gestiona a su vez desde la tensión ejercida por los condicionantes estructurales que imponen los procesos económicos, políticos y sociales complejos e interconectados (políticas migratorias, condiciones laborales en las zonas de destino, etc.) (Landolt, 2006). De acuerdo con Nina Sørensen (2007: 24), la vida de la familia transnacional «debe verse como algo influido por procesos económicos, políticos y sociales complejos e interconectados». Más allá de la agencia de los individuos, operan y constriñen factores estructurales que condicionan los patrones de movilidad de las personas.

Las transferencias económicas que se dan en el seno de la familia transnacional (principalmente las remesas monetarias) han sido ampliamente estudiadas y constituyen un pilar clave de todas las tipologías que intentan acomodar la heterogeneidad de actividades y prácticas transnacionales (Guarnizo, 1997; Portes et al., 2003). Sin embargo, existen otros tipos de vínculos, los cuales están fundamentados en la imbricación entre la esfera productiva y la reproductiva. Los vínculos de gestión del afecto y del cuidado necesariamente se

y sus colaboradores (1999) permiten delimitar el crecimiento y el alcance del fenómeno del transnacionalismo, al criticar la utilización muchas veces difusa del término transnacional. 
ven alterados por la experiencia migratoria, al disociar a los individuos de sus familias, de sus redes de amistades y de otros referentes sociales (Skrbiš, 2008). La significancia de estos vínculos se recoge en numerosos estudios que utilizan distintos términos, como "trabajo de cuidado» (caring work) (Alicea, 1999; Gonzálvez, 2005; Parella, 2007; Zontini, 2010) o «trabajo de parentesco» (kin work). Este último es definido por Di Leonardo (1992: 248) como el conjunto de actividades orientadas al mantenimiento de los lazos de parentesco dentro del grupo doméstico (visitas, celebraciones rituales, llamadas telefónicas, regalos, etc. $)^{3}$.

Asimismo, las familias afectadas por procesos migratorios, como todo grupo familiar, constituyen instituciones fuertemente marcadas por el género, lejos de la imagen unitaria de un hogar no dividido por jerarquías de poder, autoridad y recursos (Grasmuck y Pessar, 1991; Hondagneu-Sotelo, 1994; Pessar y Mahler, 2003). Por ello, hay que evitar aproximarse a la familia como entidad uniforme, sin tener en cuenta las desiguales relaciones de poder que se dan en su seno (relaciones de género, relaciones intergeneracionales), así como la asignación diferenciada de roles en los procesos de construcción de las identidades y en la reproducción del bienestar de sus miembros (Moore, 1999; Herrera, 2004, 2005).

Si bien puede afirmarse que la migración supone una renegociación de los roles de género y provoca una reestructuración de las asimetrías de género, de acuerdo con Rosas (2010: 246), se trata de modificaciones y cambios que no necesariamente «anulan la desigualdad». Es decir, la migración conlleva la potencialidad de cambio en las relaciones de género, sin que sea posible a priori establecer su ocurrencia, ni cuáles van a ser las características de este cambio, su sentido o sus resultados — si dichas asimetrías van a reducirse o, por el contrario, pueden verse incluso reforzadas- (Gregorio, 1998; Ariza, 2000; Oso, 2007).

La presente investigación pretende estudiar los ajustes transnacionales de la migración boliviana en España que tienen que ver con los roles de género, a partir del análisis de los discursos de los migrantes y sus contrapartes ubicados en distintas posiciones geográficas, sociales y simbólicas dentro de la familia transnacional. Para ello, se articulan los vínculos económicos y los de cuidado y parentesco y se incorpora el carácter relacional (entre hombres y mujeres) y situacional de la perspectiva de género.

En cuanto a la metodología, esta investigación se estructura a partir de un diseño cualitativo, bilocal, que toma a la familia transnacional como unidad de análisis. El diseño metodológico se ha basado en la técnica de la entrevista en profundidad, que permite acceder a los marcos de referencia, a las representa-

3. Skrbiš (2008) aplica a la familia transnacional el término «trabajo emocional» (emotional labor), acuñado por Hochschild (1983), para referirse al tipo de acciones que permiten el mantenimiento de la conexión afectiva a través del espacio y del tiempo, que incluyen tanto a la persona migrante como a los miembros de la familia y a otros integrantes de la familia extensa que permanecen en las zonas de origen. 
ciones simbólicas y a las estrategias, tanto de las personas migrantes como de los miembros left behind.

Han sido seleccionadas veinte familias transnacionales con migrantes de referencia instalados en Barcelona o Madrid y con el resto de miembros de la familia residiendo en Bolivia. El diseño bilocal de la investigación ha posibilitado el estudio de las interacciones intrafamiliares durante el periodo de separación, así como de los elementos de continuidad y de cambio del proceso migratorio (etapa premigratoria y postmigratoria) desde la perspectiva de los distintos miembros de la familia (Solé, Parella y Cavalcanti, 2007; Dreby, 2007).

Asimismo, de acuerdo con Baldassar (2008), la selección de los casos se ha guiado por el criterio de escoger familias con vínculos transnacionales activos, de acuerdo con la definición de Bryceson y Vuorela (2002) antes mencionada. Por consiguiente, el estudio de las relaciones familiares de género que plantea esta investigación deja de lado otras formas de reconfiguración de los vínculos familiares tras procesos migratorios que no pasen necesariamente por la estructura familiar transnacional, es decir, que no impliquen una articulación tanto de la pertenencia como de la percepción del bienestar desde una dimensión colectiva.

La primera parte del artículo ofrece una breve contextualización del flujo migratorio boliviano hacia España, desde el análisis de las dinámicas de transformación de las migraciones más recientes en relación con los patrones migratorios bolivianos «tradicionales». A continuación, se presentan las decisiones metodológicas que han guiado el diseño de la investigación cualitativa para, seguidamente, mostrar los principales resultados de la investigación en cuanto al potencial de los ajustes transnacionales de las familias a la hora de renegociar los significados y las prácticas en torno a los roles de género.

\section{El contexto migratorio boliviano hacia España: un nuevo esquema que transforma los patrones migratorios bolivianos «tradicionales»}

Bolivia se ha convertido, en los últimos años, en una de las principales zonas emisoras de emigración hacia España en plena consolidación de la España inmigrante (Cachón, 2009), especialmente como consecuencia de la exigencia de visado de entrada a la UE a partir del 1 de abril de 2007 (Whitesell, 2008; Hinojosa, 2008b; Gadea, Benencia y Quarante, 2009). Los datos recogidos por el Padrón Municipal de Habitantes del INE cifran en 202.657 las personas nacidas en Bolivia que están empadronadas en España a 1 de enero de 2011 (a 1 de enero de 2008, se alcanzó un máximo de 242.496, que ha ido disminuyendo desde entonces como consecuencia de la crisis económica). El análisis longitudinal de los datos del Padrón muestra el significativo y acelerado incremento de este flujo migratorio, especialmente a partir del año 2005.

Según los datos de la Encuesta Nacional de Inmigrantes (ENI-2007), del Instituto Nacional de Estadística, el 31,4\% de los bolivianos se emplea en el 
servicio doméstico y el 20,3\%, en la construcción (Gadea, Benencia y Quarante, 2009). Se trata de un flujo feminizado (a 1 de enero de 2011, el 58,3\% de las personas empadronadas son mujeres), con una fuerte incidencia de la irregularidad y altamente concentrado en los «nichos laborales» habituales para la inmigración económica, con precarias condiciones, tanto de empleo como de inserción residencial. Las ciudades de Madrid, Barcelona y L'Hospitalet de Llobregat concentran casi el 30\% del total de extranjeros originarios de Bolivia que residen en España a 1 de enero de 2011. Sin embargo, en los últimos años, se ha asistido a un progresivo proceso de dispersión geográfica hacia otras comunidades autónomas, con una importante presencia de bolivianos en la Comunidad Valenciana y en Murcia, así como en las provincias andaluzas de Granada, Málaga y Sevilla (Hinojosa, 2009b). La crisis económica ha tenido efectos importantes en el colectivo boliviano, al tratarse de uno de los grupos nacionales más afectado por el desempleo (Pajares, 2010). Asimismo, los extranjeros de nacionalidad boliviana son los que han experimentado, junto con los ecuatorianos, un mayor crecimiento proporcional de salidas de España entre los años 2006 y 2008 (casi se han triplicado), fechas que coinciden con el inicio de la crisis, según los datos ofrecidos por la Estadística de Variaciones Residenciales del INE (Pajares, 2010).

La mayor parte de los migrantes bolivianos que residen en España proceden de los departamentos de Cochabamba y Santa Cruz de la Sierra. Sin embargo, en la medida que se ha ido consolidando este flujo migratorio, los lugares de origen se han diversificado y se han incorporado también las zonas rurales de los valles, el oriente y el altiplano (Hinojosa, 2009b). España constituye el segundo país de destino de la migración boliviana, tras Argentina, según los registros de vacunación de fiebre amarilla del departamento de Cochabamba para el año 2006 (Hinojosa, 2008b). Desde el año 2002 hasta abril de 2007, alrededor de 70.000 personas han emigrado de la región de Cochabamba con destino a España, lo que supone cerca del 10\% de la población (Hinojosa, 2009a).

Los resultados de una encuesta en Cochabamba — realizada en las filas de vacunación entre el 5 y el 11 de mayo de 2006 - constatan que se trata principalmente de procesos de «segunda migración» por parte de personas que han protagonizado con anterioridad migraciones internas desde el campo hacia zonas periurbanas o urbanas. Por consiguiente, de acuerdo con Hinojosa (2009b), se trata de personas con un intenso arraigo rural comunitario, aunque procedan de zonas urbanas metropolitanas. La urbanización de las emigraciones junto con su feminización, según el autor, son dos aspectos definitorios de las actuales dinámicas migratorias bolivianas (Hinojosa, 2009b)

Sin embargo, estos recientes flujos migratorios hacia España deben situarse dentro de la dilatada experiencia histórica de movilidad socioespacial de los bolivianos dentro y hacia el exterior del país (Hinojosa, 2009a). España como sociedad de destino configura un nuevo modelo de desplazamiento que no es comprensible si no se analiza dentro de las coordenadas de las estrategias de supervivencia de la sociedad boliviana, a través de arraigadas prácticas familiares 
y comunitarias de movilidad que diversos autores han denominado «cultura de la movilidad» (De la Torre, 2006; Hinojosa, 2009a) ${ }^{4}$.

Durante las últimas décadas del siglo pasado, los procesos migratorios internacionales de Bolivia se focalizan principalmente en países fronterizos como Argentina y Brasil, sin olvidar Estados Unidos como destinación destacada. Argentina, en concreto, ha sido y sigue siendo el principal destino y es precisamente Argentina la procedencia de muchos de los migrantes de la primera oleada de migración boliviana hacia España ${ }^{5}$, principalmente durante los años 2001 y 2002 (Hinojosa, 2009b). Las causas de este cambio de orientación son diversas (Bastia, 2007; Gadea, Benencia y Quarante, 2009): las crisis económicas por las que atravesaron los países latinoamericanos que habían servido de destino de las corrientes interregionales (la devaluación del peso en Argentina en 2001 marca un punto de inflexión); los atentados del 11 de septiembre en Nueva York y la redefinición de las políticas de seguridad nacional en referencia a las fronteras; sin olvidar la fuerte demanda en España de mano de obra para el trabajo doméstico asalariado y otros servicios poco cualificados. Además, de acuerdo con Hinojosa (2009a), las redes migratorias que salen de Argentina también desempeñan un papel clave en la iniciación y amplificación de la emigración boliviana hacia España. Un importante número de migrantes bolivianos que regresan de Argentina invierten sus ahorros para emigrar hacia España (Bastia, 2007).

Como ya se ha mencionado, uno de los principales cambios lo constituye el hecho de que, por primera vez en la larga trayectoria migratoria del país, se produce una marcada feminización de los flujos migratorios que altera las relaciones de género de forma sustancial (Cortes, 2004). Tal feminización es consecuencia de las transformaciones en los mercados de trabajo de los países de destino, que se han convertido en destacados reclutadores de mano de obra femenina para llevar a cabo las tareas de cuidado y otras ocupaciones de bajo salario, principalmente en el sector servicios (Parella, 2007). Las dinámicas de feminización de las migraciones internacionales se enmarcan en las dinámicas de participación de las mujeres en la producción para un mercado global (Sassen, 1984; Benería, 2008), así como en la globalización del trabajo de cuidado (globalization of care work) (Zimmerman et al., 2005). Es en este contexto que debe situarse la salida de mujeres bolivianas hacia España y hacia Estados Unidos (Zarembka, 2003).

Otra transformación a tener en cuenta es la imposibilidad de mantener los patrones circulares que habían caracterizado las migraciones interregionales (hacia Argentina y Brasil). La exigencia de visado a los inmigrantes bolivianos

4. Según los datos del informe La situación de migrantes en Bolivia (2006), elaborado por el Capítulo Boliviano de Derechos Humanos Democracia y Desarrollo, más de 2 millones y medio de personas residen fuera del país, por encima del $30 \%$ de la población total (Hinojosa, 2008a: 81).

5. Con tendencia a expandirse también hacia otros países europeos (Italia, Reino Unido, Suiza) e incluso a destinos como Japón e Israel (Hinojosa, 2008a). Esta tendencia a la dispersión dentro de Europa se observa en la actualidad con especial intensidad, a consecuencia de la destrucción de empleo en España. 
en Europa y el carácter reciente del flujo migratorio hacia España se traduce en una fuerte incidencia de la irregularidad. Ello impide a muchos de estos migrantes poder llevar a cabo «idas» y "venidas» entre España y Bolivia, ante las dificultades para volver a entrar. La imposibilidad de diseñar patrones de movilidad más circulares impacta de forma rotunda en las estructuras familiares y en las estrategias a la hora de reacomodar las relaciones, tanto de género como entre las generaciones.

Las migraciones de bolivianos a Argentina, en cambio, sí han permitido el retorno periódico a los lugares de origen, por cuanto se dan en un contexto legal de mayor flexibilidad de ingreso, de regreso y de residencia (Dandler y Medeiros, 1991). De ese modo, los desplazamientos espaciales se convierten en una estrategia más para las unidades familiares rurales y urbanas, dentro de un amplio abanico de actividades económicas diversificadas y estrategias de autoempleo ligadas al sector informal, en un marco de amplias redes sociales (Dandler y Medeiros, 1991). Se trata de desplazamientos que autoras como Genevieve Cortes (2004) han abordado como parte de un "ciclo migratorio", que conjuga el itinerario de migración — de ida y venida - y su relación con el ciclo vital del individuo, las familias y sus proyectos de vida. Generalmente, es el varón quien emigra - habitualmente a Argentina - y la unidad familiar se consolida a distancia, desde una división sexual del trabajo.

Aunque las mujeres en Bolivia conciben la identidad de género dentro de un enfoque tradicional (Peña et al., 2003), el valor económico que adquieren a temprana edad y la autonomía que les confiere el carácter más igualitario y bilateral del sistema de parentesco andino, han supuesto un freno a su emigración internacional. Mientras los varones emigran por una necesidad laboral, vinculada al proceso de creación de un nuevo hogar, las mujeres son un bien cotizado en origen que contribuye al ingreso familiar. Por consiguiente, tienen menos motivos para protagonizar una migración laboral de tipo temporal o circular (Balán, 1990). Según los datos de Balán (1990), sólo se trasladan a Argentina mujeres solteras o bien mujeres que se han visto sometidas a grandes tensiones familiares.

Aún así, el peso de las mujeres de origen boliviano en Argentina, en base a los datos de los censos, se ha ido incrementado progresivamente. Ello está ligado a los procesos de reagrupación familiar y al aumento de la migración de mujeres jóvenes y solteras. El índice de masculinidad se reduce de 148 en el año 1960 a 101 en el año 2001 (Baby-Collin, Cortes y Sassone, 2008: 139). Las mujeres que emigran a Argentina suelen trabajar de empleadas domésticas o como vendedoras callejeras o artesanas (Magliano, 2007). Las mujeres solteras, generalmente, se emplean en el sector textil, como cosedoras o tejedoras, a las órdenes de otras mujeres bolivianas y casi siempre vinculadas al «sector informal» (Balán, 1990; Dandler y Medeiros, 1991; Bastia, 2007). Los hombres, en cambio, se concentran principalmente en la construcción, la agricultura y las fábricas de ladrillos (Magliano, 2007).

Con el cambio de rumbo de los flujos y la feminización de la migración hacia España, se confrontan los imaginarios de género que habían predominado hasta entonces, basados en la organización de proyectos migratorios 
internacionales eminentemente masculinos (Pedone, 2008). Se trata de una migración femenina que ya no incluye preferentemente a mujeres solteras, sino que se construye como estrategia familiar de supervivencia. Haciendo extensivos los resultados de los trabajos de Pedone (2008) para el caso ecuatoriano, las mujeres bolivianas, a partir de la emigración a España, se convierten en las principales proveedoras materiales del grupo doméstico, en aras de mejorar las condiciones de trabajo del cónyuge y lograr el acceso de los hijos a una educación de mayor calidad. Sin duda, la participación activa de la mujer en el desplazamiento de la población boliviana, como primer eslabón de la cadena migratoria, conlleva profundas transformaciones a nivel familiar y ha provocado la emergencia de una alarma social respecto a los cambios que ello supone dentro de las estructuras familiares involucradas en el hecho migratorio.

\section{Metodología de la investigación}

Sin duda, el diseño metodológico de esta investigación, bilocal y de corte cualitativo, permite analizar los elementos de continuidad y de cambio del proceso migratorio (etapa premigratoria y posmigratoria) desde la forma cómo se percibe por parte de los distintos miembros de la familia, a su vez ubicados en distintas posiciones de poder dentro del grupo familiar. Asimismo, por cuanto se concibe el género como proceso relacional, que toma en cuenta tanto a los hombres como a las mujeres, se supera el sesgo hacia solo las experiencias femeninas que presentan muchas de las investigaciones que abordan las construcciones de género y el proceso migratorio (Hondagneu-Sotelo, 2000; Rosas, 2010).

Puesto que esta investigación toma la familia como unidad de análisis, una de las decisiones metodológicas clave ha sido identificar qué tipo de estructura familiar transnacional es la más adecuada para el caso boliviano; en el sentido de optar por considerar estructuras nucleares o, por el contrario, patrones de familia extensa. De acuerdo con las investigaciones de Dandler y Medeiros (1991: 21), las unidades familiares andinas se componen, en su mayor parte, de una sola familia nuclear, a pesar de que estén enlazadas por extensas relaciones familiares y que la familia nuclear se inserte en una estructura organizativa más amplia. Las familias compuestas por padres e hijos casados suelen vivir en el mismo grupo de casas y participan en intercambios de productos y de trabajos personales, si bien cada unidad familiar controla sus propios recursos (Balán, 1990: 278). En la misma línea, Leonardo de la Torre (2006: 126), para el caso boliviano, define la familia transnacional como la que "participa del fenómeno migratorio a través de uno o más de los miembros de la unidad familiar nuclear, compuesta por padre, madre, hermanos o por hijos, esposo o esposa» ${ }^{6}$.

6. A pesar de esta definición operativa, nuestra investigación toma en cuenta el hecho de que las prácticas transnacionales (remesas, demostraciones de solidaridad familiar y comunitaria migrante, etc.) se gestionan no sólo desde la necesaria vinculación nuclear con los familiares migrantes, sino también en un marco de parentesco ampliado (Balán, 1990: 278; De la Torre y Alfaro, 2007). 
La selección de los casos se ha guiado a partir de tres criterios: el tipo de estructura familiar, la región de origen (Cochabamba, La Paz, Oruro y Santa Cruz) y el lugar de residencia del migrante de referencia en España (Madrid o Barcelona, los principales destinos en términos cuantitativos). En cuanto al tipo de estructura familiar, el estudio contempla cuatro situaciones de familia transnacional, a partir de la adaptación de las tipologías de Oso (1998) y Parreñas (2001):

1. Familia nuclear en la que emigra la mujer.

2. Familia nuclear en la que emigra el varón.

3. Familia monoparental en la que emigra uno de los dos progenitores.

4. Familia nuclear en la que emigran los hijos adultos ${ }^{7}$.

A continuación, se detallan las etapas que ha seguido el trabajo de campo:

- Primera etapa (enero-agosto de 2008). Selección de los migrantes de referencia en Madrid y Barcelona. Se construye un casillero tipológico de veinte familias y se realizan las entrevistas en profundidad a los veinte migrantes.

- Segunda etapa (agosto 2008-junio de 2009). Contactos con los miembros de la familia transnacional de cada migrante de referencia que permanecen en Bolivia: Cochabamba, Santa Cruz, Oruro y La Paz. Las entrevistas en profundidad se han realizado o bien al cónyuge - para el caso de estructuras nucleares-, o bien a un hijo o a una hija mayor de dieciséis años, o bien al padre o a la madre, en el caso de adultos no emancipados del hogar familiar en el momento de emigrar.

Abordar los impactos en las relaciones de género plantea importantes retos a la hora de construir los indicadores adecuados para medirlos, tanto si se trata de diseños metodológicos cualitativos como cuantitativos. Por un lado, debe tenerse en cuenta la dimensión longitudinal, por cuanto los impactos en los roles de género son dinámicos y procesuales, no necesariamente sostenidos a lo largo del tiempo. Asimismo, hay que considerar las formas de subordinación de género de las que parten las mujeres bolivianas a tenor de su origen étnico, de su pertenencia de clase y de su posición dentro del proceso migratorio. Por ello, en esta investigación se han utilizado dimensiones de análisis que permiten, por un lado, dar cuenta de los cambios en las relaciones de género y, por el otro, abordar tanto la perspectiva discursiva del varón como la de la mujer:

1. Cambios en las percepciones sobre los roles familiares de género en relación con el momento premigratorio.

2. Cambios en las prácticas que tienen que ver con la división sexual del trabajo en relación con las prácticas premigratorias.

7. Para esta investigación, se ha descartado la forma de familia transnacional en la que emigran ambos cónyuges y los hijos permanecen a cargo de otros miembros de la familia extensa (generalmente, las abuelas). 


\section{Arreglos y formas de gestión de los vínculos transnacionales y sus impactos en los roles de género: el caso boliviano}

\subsection{Los condicionantes de género en los proyectos migratorios}

Un aspecto fundamental para el análisis de los ajustes transnacionales que tienen que ver con los roles de género es considerar los proyectos migratorios concebidos en sí mismos desde parámetros de género. Los condicionantes de género intervienen en los proyectos migratorios, tanto de los varones como de las mujeres, ya que ambos son sujetos "generizados» (HondagneuSotelo, 2000). Cuando son los varones quienes emigran en primera instancia, la decisión se plantea en clave familiar y supone un reforzamiento de la representación social que asocia la ejecución del proyecto migratorio internacional a la figura del varón "cabeza de familia» (el «hombre de la casa»). Se asume que la emigración del "padre» va a generar una menor disrupción dentro del grupo familiar (Pedone, 2004). Sin embargo, son muchas las investigaciones que vinculan la feminización de las migraciones con la demanda de empleadas domésticas en las sociedades de destino, en el marco de la división internacional del trabajo reproductivo (HondagneuSotelo, 2000; Parreñas, 2001). Así ocurre para el caso boliviano (Parella y Cavalcanti, 2010).

La certeza de que va a resultar más fácil en España encontrar empleo para las mujeres que para los varones, junto a la mayor capacidad de ahorro que supone el servicio doméstico - máxime si se trata de la modalidad de «internas»—, son otros de los detonantes esgrimidos por parte de las familias entrevistadas. Se trata de proyectos bidireccionales (de ida y regreso), construidos desde el retorno, que describen situaciones de movilidad femenina como resultado de las estrategias de los hogares, en las que, de forma consensuada, las mujeres asumen la responsabilidad económica de sus familias (marido, hijos y otros familiares) (Oso, 2007).

Cuando se trata de mujeres que son jefas de hogar, por cuanto ya habían asumido el papel de sustentadoras económicas principales antes de emigrar, es precisamente esta situación la que motiva su proyecto migratorio. Algunas entrevistadas atribuyen su proyecto migratorio a la falta de corresponsabilidad masculina en el pago de los gastos de la familia y a la ausencia de una paternidad responsable. Los padres de sus hijos, con los que no convivían, se presentan como actores pasivos en dicho proceso (Rosas, 2010).

Asimismo, Hondagneu-Sotelo (1994) y Rosas (2010) documentan, para el caso de la emigración mexicana a EE.UU, que algunas mujeres emprenden proyectos migratorios para cambiar relaciones «opresoras» con sus esposos y consumar así procesos de separación o divorcio que les permitan evitar tener que afrontar un conflicto directo. Nuestro trabajo de campo no ha permitido identificar este tipo de discursos como detonantes explícitos de la decisión de emigrar (etapa premigratoria). Sin embargo, sí aparecen en las entrevistas testimonios de mujeres que encuentran en la posmigración motivaciones para llevar a cabo esta ruptura. 
Cuando se trata de mujeres que emigran de forma "pionera", seguir cubriendo el rol de la reproducción social durante el periodo de separación requiere de muchos arreglos en el entorno familiar (Pedone, 2008). La tensión entre el rol productivo y el rol reproductivo que la migrante asume se acrecienta con la ausencia. La atribución del trabajo reproductivo a la mujer implica mayores esfuerzos de redistribución y aumento de cargas de trabajo y responsabilidades a otras mujeres cuando es ella la que se desplaza a otro país (Vidal et al., 2002). Por ello, desde parte del discurso político, académico y mediático, se señala la separación de la familia cuando la "pionera» es una mujer, como el principal detonante de efectos negativos, tales como el incremento del número de divorcios, alcoholismo masculino, embarazos precoces de adolescentes, bajo rendimiento escolar de los niños, delincuencia, drogas e incluso alta incidencia de suicidio infantil o de abusos sexuales (Hochschild, 2003; Parella, 2007; Sørensen, 2007).

Se estigmatiza a las «mujeres de la migración» a base de enfatizar las consecuencias devastadoras que se atribuyen al «abandono del hogar» ${ }^{8}$. Este discurso, de acuerdo con las tesis que sostiene Parreñas (2001, 2003), tiene poco que ver con la situación real de los hijos, y sí mucho en cambio con la necesidad de renegociar las relaciones de género en un contexto de migración femenina que pone en jaque a los pilares del patriarcado. De acuerdo con los trabajos de Wagner (2008: 335) para el caso ecuatoriano, se trata de estereotipos relacionados con las transformaciones de género en el contexto no solo transnacional, sino también local. Estos estereotipos son reflejo de discursos sancionadores, de tipo moral, que atribuyen la falta de cumplimiento del «rol femenino materno" por parte de las mujeres que trabajan fuera del hogar. Para los varones, en cambio, si bien la migración también supone alteraciones del significado y el ejercicio de la paternidad, suele implicar un reforzamiento del rol de sustentador económico y le otorga prestigio social (capital simbólico) (Pedone, 2004).

De acuerdo con INSTRAW (2005), los proyectos migratorios muestran «una gran plasticidad y se moldean en función de una multiplicidad de variables que van surgiendo a lo largo de la trayectoria migratoria de cada caso particular» (2005: 62). Así se ha constatado para el caso de la migración boliviana residente en España. Si bien nuestro trabajo de campo recoge principalmente proyectos inicialmente diseñados a modo de estancias más o menos breves en destino, supeditadas al logro de los objetivos económicos planeados (pago de estudios, adquisición de vivienda, etc.), con el tiempo pueden llegar a transformarse en proyectos más largos, que impliquen la reagrupación de la familia o de una parte, o simplemente postergar el retorno. Tales cambios no siempre llegan de manera consensuada por parte de todos los miembros del grupo familiar y a menudo generan conflicto.

8. Así lo muestran los trabajos de Solfrini (2005), Wagner (2008) y Pedone (2008) para el caso ecuatoriano, de Vidal et al. (2002) para la migración autónoma de mujeres mexicanas a USA, o las investigaciones de Parreñas (2000) para el caso filipino. 


\subsection{Cambios en las percepciones sobre los roles familiares de género a raíz del proceso migratorio}

Tal como señalan Tienda y Booth (2008), la migración puede suponer tanto pérdidas como ganancias para las mujeres, lo que se traduce en un proceso permanente de reestructuración de asimetrías que va redefiniendo las relaciones sociales y que afecta tanto a las personas que emigran como a las que permanecen en las zonas de origen. Por otra parte, otra dificultad es pensar indicadores con vocación de universalidad, por cuanto lo que, para un contexto determinado, puede resultar emancipador, para otras realidades sociales puede significar todo lo contrario. De acuerdo con Magliano y Romano (2011), plantear la migración como «experiencia de independencia» y de "mayor igualdad» obliga a preguntarse, por un lado, a qué modelo de mujer autónoma se hace referencia (p. 53). Por el otro, en qué medida no se está reproduciendo una visión etnocéntrica, que de forma automática vincula la migración hacia sociedades «modernas» al empoderamiento femenino (Gregorio, 1998), desde discursos basados en el «patrón de poder colonial» (Quijano, 2005).

Qué duda cabe que medir la emancipación femenina desde el acceso al trabajo remunerado tras la migración no es un indicador adecuado cuando se trata de mujeres laboralmente activas en el país de origen. Algunas desempeñaban puestos cualificados y en la sociedad de destino se enfrentan a la «inconsistencia de estatus» que conlleva su acceso a segmentos laborales poco cualificados y de escasa valoración social — como es el servicio doméstico, por ejemplo. Por otra parte, para las mujeres left behind, aunque sigan laboralmente inactivas, el control de las remesas puede convertirse en un elemento favorecedor de su empoderamiento9. En definitiva, no es posible generalizar sobre el impacto de los fenómenos migratorios en las relaciones de género, por cuanto éstos dependen de los contextos premigratorios y postmigratorios, así como de las diferencias en las pertenencias de clase o del origen étnico a la hora de explicar el tipo de modelo familiar y el peso de los componentes patriarcales (Magliano, 2007) ${ }^{10}$.

Los resultados de la investigación muestran, ante la emigración masculina, un discurso que mantiene vigente el rol del varón sustentador como «figura de autoridad", así como el de la mujer responsable de la reproducción social. El rol del varón puede no verse debilitado con la distancia, gracias a la posibilidad que ofrecen las nuevas tecnologías a la hora de mantener su capacidad de decisión sobre, por ejemplo, cómo gastar el dinero que el migrante envía. Sin embargo,

9. Los resultados de la investigación de Rosas (2008) con mujeres veracruzanas (México) cuyos cónyuges han emigrado a Chicago (Estados Unidos), muestran que ellas acceden a nuevos espacios simbólicos y materiales posibilitados por la migración.

10. Robert (2009) constata diferencias étnicas en cuanto a los impactos en las relaciones de género, a partir del estudio de dos zonas emisoras distintas dentro de República Dominicana. Mientras en Vicente Noble se detecta un patrón étnico y cultural afrodescendiente, con una generalización de la jefatura de hogar femenina, inestabilidad marital y ausencia del modelo male breadwinner; en la Placeta, en cambio, predomina la población mestiza y un componente patriarcal mucho más determinante en las relaciones familiares. 
la situación transnacional a la que se enfrenta la familia sí puede conllevar cambios en los roles femeninos para las mujeres left behind. Ante la perspectiva del regreso del esposo, en algunos casos la mujer percibe la recomposición de las relaciones de pareja desde la proximidad y el contacto diario como un proceso complicado; por cuanto se asume que el periodo de separación le ha permitido a ella ganar autonomía y libertad de movimiento. Así se pronuncia Raquel ante el inminente retorno de su esposo, Samuel:

Ahora que va a volver y ya es definitivo, siento un poquito de temor, porque yo me he acostumbré a vivir sola, a tomar mis decisiones. Ahora no sé, sólo digo: «iAy, Diosito lindo, solamente dame paciencia!». Una amiga me comentó que su esposo se fue durante tres años a Chile, a hacer una especialidad; y que cuando volvió todo fue terrible. «Ahora, Raquel, que tu esposo va a volver», me dice, «no cometas el mismo error que yo he cometido, tenle más paciencia, no le des lucha desde el primer momento; cuando llegó mi esposo cada uno quería hacer las cosas de una forma, nos hemos gritado, hemos llegado a los golpes y nos hemos separado, un año y medio.» (Raquel, esposa de Samuel, La Paz)

Sin embargo, como era de esperar, es ante la emigración femenina pionera donde se han identificado mayores impactos en los roles familiares de género es ante la emigración femenina. Los trabajos de Parreñas (2005) ponen de manifiesto, para el caso filipino, que las estructuras familiares transnacionales conllevan contradicciones de género. La emigración femenina pionera, como ya se ha comentado, genera fisuras en torno al papel tradicional de la «esposa» $\mathrm{y}$ "madre», al tiempo que los cónyuges varones mantenidos por las remesas femeninas se enfrentan al cuestionamiento de la masculinidad tradicional (Oso, 2007).

La mujer, por lo general, debe hacer frente al dolor por la separación y a la estigmatización social por no estar junto a sus hijos. La estigmatización social focalizada en la "ausencia femenina" se refleja en el discurso de los mismos maestros y profesionales en las escuelas, que atribuyen a la emigración femenina el bajo desempeño escolar de los niños (Fe y Alegría, 2010). Expresiones del tipo: "Se cayó porque su mamá no está aquí», «No hizo la tarea porque su mamá está en España», "Se ríe porque su mamá no está» son habituales en las escuelas, según se menciona en alguna de las entrevistas realizadas.

Sin embargo, al mismo tiempo, la mujer migrante se enfrenta a mayores cotas de emancipación una vez en España y a nuevos ámbitos de socialización. Así lo han manifestado algunas de las entrevistadas. Tal emancipación se verbaliza no sólo de forma explícita, sino principalmente mediante alusiones a un mayor empoderamiento en cuanto al grado de control sobre el proyecto migratorio. Dicho empoderamiento se expresa ya sea planteando la reagrupación familiar en España como un nuevo objetivo, a pesar de que el proyecto migratorio inicialmente no la contemplaba.

Para las mujeres que han emigrado, se percibe el logro de prestigio no tanto a partir del tipo de inserción socioeconómica lograda (fundamentalmente en el trabajo doméstico asalariado, una actividad absolutamente denostada en Bolivia), sino en base al hecho de poder ejercer de principales sustentadoras del 
grupo familiar. Se trata de un discurso que combina el logro económico para sus familiares con una forma activa de construcción de su propia emancipación, utilizando la competencia adquirida a través de la migración (Baby-Collins, Cortes y Sassone, 2008). Al respecto, Rosa, una mujer cochabambina que reside en Barcelona, narra los cambios que ha experimentado, tanto a nivel de autonomía económica como de autonomía personal:

Sí que ha habido cambios, porque, quieras o no, al estar sola, lamentablemente te acostumbras a estar sola, allá en Bolivia, quieras o no, no sé si es el machismo siempre el hogar es del jefe de familia, manda, y económicamente él siempre estaba en todo, entonces uno ahí no se siente libre pudiente económicamente, quieras o no es un cambio para la mujer boliviana. Porque allá la mujer es para la casa, muy pocos dejan que trabajen, siempre está en la casa con los hijos, no tienen ningún ingreso y siempre dependen del marido y al estar aquí eso se rompe totalmente claro, ya quien dispone y hace es la mujer (Rosa, Barcelona)

Para el varón, en cambio, la emigración de sus parejas supone tener que lidiar con el estigma de "hombre mantenido», alguien que no ejerce su masculinidad según los cánones tradicionales. Estos «nuevos» patrones migratorios feminizados sin duda exigen flexibilizar la masculinidad, ante las dificultades para seguir cumpliendo con éxito el rol de varón proveedor (Rosas, 2010). Ángel, un varón de cuarenta y dos años que reside en Cochabamba con sus dos hijos adolescentes, admite que "para mí ha sido difícil, porque cuando yo me casé con ella le prohibí que trabajara. Vengo, creo, de una familia muy machista y, obviamente, el hombre es el que tiene que mantener y todo eso". No todos los hombres cuentan con las mismas estrategias a la hora de hacer frente a las presiones de género. Nuestro trabajo de campo pone de manifiesto que los recursos educativos y las pertenencias de clase marcan diferencias. Mientras algunos son capaces de «ignorar» las presiones sociales que reciben; otros refuerzan el control y la presión sobre la mujer desde la distancia, a través de un discurso de reproche y estado de alerta frente a posibles y eventuales cambios de sus parejas tras la migración, que puedan cuestionar su papel de «hombre de la casa» (la forma de hablar, de vestir, etc.).

Por último, la percepción de fragilidad a la hora de gestionar la relación de pareja desde la distancia aparece tanto en el discurso de los varones como de las mujeres. Se asume que la migración conlleva mayores posibilidades de iniciar un romance para las personas que están lejos de sus familias. Lógicamente, el nivel de comunicación que exista dentro de la pareja previamente y durante el proceso migratorio es fundamental para hacer frente a tales riesgos, tal como ponen de manifiesto las personas entrevistadas, tanto varones como mujeres. Sin embargo, incluso así, las dudas suelen estar presentes en los discursos de las personas entrevistadas.

En algunos casos, los rumores y la incertidumbre acerca de la fidelidad del otro no sólo «siembran las dudas», sino que pueden conllevar la fragmentación de la relación de pareja con el tiempo. Así ha sucedido para una de las parejas entrevistadas que, si bien no ha formalizado todavía la separación legal, se asume 
como «algo inevitable», por ambas partes, atribuible a la distancia. A modo de ejemplo ilustrativo, el trabajo de campo de nuestra investigación recoge algunas experiencias de parejas que se han roto a raíz de la distancia, así como referencias a expresiones del tipo "amor a cuatro», para designar situaciones en las que tanto el miembro de la pareja que emigra como el que permanece tejen nuevas relaciones amorosas para hacer frente a la soledad que conlleva el tiempo de separación.

\subsection{Cambios en las prácticas que tienen que ver con la división sexual del trabajo en relación con las prácticas premigratorias}

En cuanto a los cambios en las prácticas que tienen que ver con la división sexual del trabajo, no se producen alteraciones significativas cuando emigra el varón, por cuanto sigue correspondiendo a la mujer la responsabilidad en el trabajo doméstico y familiar. Sin embargo, el trabajo de Pribilsky (2004), basándose en el caso de los migrantes varones ecuatorianos en Nueva York que han "dejado» a su mujer en Ecuador, muestra la necesaria redefinición de los roles de género que también se produce ante la ausencia del varón. No sólo los varones desarrollan en la sociedad de destino funciones que tradicionalmente eran consideradas femeninas (como cocinar o limpiar), sino que las mujeres adquieren nuevas responsabilidades además de encargarse de las remesas y pasan a asumir algunas tareas que solían hacer los hombres antes de emigrar.

Cuando emigra el varón, las mujeres left behind ven reforzado su papel en la gestión de la economía familiar (gracias a la recepción de las remesas), al tiempo que recae en ellas la responsabilidad de mantener la cohesión familiar, a través de la reconstrucción discursiva del proyecto migratorio del «padre ausente» como algo compartido y beneficioso para todo el grupo familiar. Además, algunas de las mujeres entrevistadas en nuestro estudio adquieren funciones que antes correspondían al cónyuge varón, como, por ejemplo: negociar con los acreedores o recoger a los hijos de noche tras sus salidas nocturnas (véase el testimonio de Ana María) ${ }^{11}$ :

Lo que ha cambiado es la responsabilidad. Por ejemplo, antes ella [la hija] salía, ¿no? y él iba a recogerla, de noche. Yo no me preocupaba porque él, el varón. Y ahora ella sale, para mí es una preocupación, me da miedo. Entonces ya no es esa tranquilidad que tenía antes. Eso ha cambiado. (Ana María, esposa de Ernesto, Oruro)

En cualquier caso, los resultados de nuestro estudio confirman que el «trabajo de parentesco" (kin work) de las mujeres que permanecen en origen es fundamental para el mantenimiento del fathering como figura simbólica y de

11. A conclusiones análogas nos remiten los resultados de la investigación de Rosas (2008) con mujeres veracruzanas (México) cuyos cónyuges han emigrado a Chicago (Estados Unidos), al constatar que ellas acceden a nuevos espacios simbólicos y materiales posibilitados por la migración: flexibilización del control por parte de los esposos, una mayor presencia en el ámbito público y disponibilidad de dinero. 
autoridad. El caso de Ernesto y Ana María así lo pone de manifiesto. Si bien las llamadas de Ernesto a su familia desde Barcelona se producen a diario y a través de las conversaciones telefónicas gestiona la vida familiar (viajes escolares de sus hijos, salidas de fin de semana, etc.), es gracias a la colaboración de su esposa que logra mantener su autoridad como padre. Es ella la que "promueve» que siga siendo Ernesto a quien sus hijos deben pedir permiso para poder salir con sus amigos, o el que decide cuándo hay que infringir algún castigo.

Ante la emigración femenina, los trabajos de Parreñas (2005: 332) evidencian cómo las contribuciones económicas de las mujeres filipinas que habían emigrado a los Estados Unidos no implican una reconfiguración de la división sexual del trabajo dentro de la familia. Los denominados househusbands raramente se ocupan del trabajo doméstico y familiar. Para el caso boliviano, nuestros resultados muestran que, cuando es la mujer la que emigra, se asiste a un incremento de la implicación del varón en las tareas reproductivas, aunque siempre en calidad "de apoyo» a otras mujeres de la familia, generalmente las «suegras». De ese modo, o bien son las abuelas las que se trasladan al hogar familiar, o bien son los hijos y el esposo los que se instalan en la vivienda de sus suegros o de sus padres.

Cuando la persona migrante encabeza un hogar monoparental, los niños que permanecen en Bolivia suelen quedar a cargo de otros miembros de la familia extensa (generalmente, abuelas o hermanas mayores), que son, a su vez, las personas que gestionan las remesas. En estos casos se confirma que los impactos en los hijos dependen principalmente de la calidad de las redes familiares de las que disponen las familias.

Efectivamente, las mujeres desempeñan un papel clave como pivotes en el mantenimiento de los lazos familiares, a pesar de la distancia física que separa a sus miembros (Baby-Collins, Cortes y Sassone, 2008; Zontini, 2010). Cuando ellas emigran, además de la función económica y productiva que asumen (envío de remesas), son las principales articuladoras de los espacios sociales transnacionales a través de su trabajo reproductivo cotidiano (Pedone, 2004). Las mujeres son centrales en la organización socioespacial de la migración y, en definitiva, en la asunción de los distintos rituales de socialización a fin de mantener la cohesión del grupo familiar (Alicea, 1999; Pedone, 2004) ${ }^{12}$.

La emigración de las mujeres transforma, reorienta e impacta en los hijos que permanecen en origen en mayor medida que la masculina. Así se confirma para los resultados de nuestro trabajo de campo. Los efectos negativos ante la ausencia de la madre son mencionados de forma explícita por algunos testimonios y están estrechamente vinculados a la edad de los hijos durante el periodo de separación. Los efectos oscilan desde un descenso del rendimiento académico (especialmente durante los primeros años), hasta un rechazo hacia

12. Sin embargo, Baldassar (2008) muestra que la dimensión de «mantener el contacto» que requiere el kin-work definido en los términos de Di Leonardo (1992), cada vez la asumen en mayor medida los hombres, particularmente los más jóvenes, dado su mejor manejo de las nuevas tecnologías de la comunicación (correo electrónico y SMS). 
la madre como respuesta al sentimiento de «abandono». La capacidad de la madre de mantener el contacto desde la distancia es fundamental a la hora de modular los efectos de la separación, aunque no siempre es suficiente para compensar estas funciones «afectivas» que el padre no se ve capaz de asumir.

Para Dulia y Gastón, los impactos negativos que tiene en sus hijos la emigración de la madre afectan especialmente a los más pequeños que, por su edad, no pueden todavía comprender qué ha motivado su ausencia. Dulia asume que lo más duro para ella es tener que aceptar que los más pequeños no entienden por qué se ha ido. El esposo, Gastón, maestro de escuela, coincide con su esposa al afirmar que sus hijos echan de menos a su madre y que difícilmente él puede reemplazar su papel.

No, es que yo lo comprendo y mis hijos no han comprendido por qué estoy como estoy. Los chiquititos, ellos no comprenderán nunca que estoy aquí, ellos no saben la razón de mi realidad, entonces es una gran diferencia. (Dulia, Madrid)

Eso mucho tiene que ver con quién se haya quedado. Aparentemente están tranquilos, pero emotivamente están esperando que les pasen un bracito, una caricia, por la cabeza. Eso se nota en la escuela. A veces, no tengo mucho tiempo, estamos corriendo como locos. Mi hija es la que más se queda. A veces le dicen «Mami». Entonces eso da a entender de que están buscando a su madre, un apoyo. Entonces yo, como estoy como un loco, a veces, a un lado a otro lado, no hay ese apoyo. (Gastón, esposo de Dulia, Oruro)

La figura 1 recoge una síntesis de los principales impactos y ajustes identificados sobre los roles familiares de género, dependiendo de si se trata de una emigración pionera masculina o femenina.

Figura 1. Impactos de la emigración en los roles familiares de género: percepciones y prácticas

\begin{tabular}{ll}
\hline Emigración masculina & Reforzamiento del rol de «varón sustentador». \\
& Percepción de mayor autonomía por parte de las mujeres left \\
& behind. \\
& Las mujeres asumen «nuevas» responsabilidades ante la \\
& ausencia masculina. \\
\hline Emigración femenina & Discurso «emancipador» por parte de las mujeres migrantes \\
& con cónyuge en Bolivia. \\
& Discurso de continuidad para las mujeres jefas de hogar: \\
& ejercen de sustentadoras durante las etapas premigratoria y \\
& postmigratoria. \\
& «Hombre mantenido» como categoría estigmatizadora. \\
& Incremento de la implicación masculina en las tareas reproduc- \\
& tivas, aunque desde el apoyo a «otras mujeres» (generalmente, \\
& sus suegras).
\end{tabular}




\section{Conclusiones}

Con el auge de la "perspectiva transnacional de la migración», la familia transnacional - a pesar de la dificultad que entraña su delimitación- se consolida como unidad de análisis para el abordaje académico de las dinámicas migratorias y de las reconfiguraciones de las relaciones de género e intergeneracionales que de ellas se derivan. Desde una perspectiva de género, esta forma de organización social no sólo permite captar las transferencias económicas familiares y la gestión y los impactos de las mismas (las remesas monetarias). Existen otros tipos de vínculos que se dan en su seno, los de gestión del afecto y del cuidado y el trabajo de parentesco, que son la base de la imbricación entre las actividades de producción y reproducción y que la investigación hasta hace muy poco tiempo había relegado a un segundo plano, a pesar de constituir una de las principales bazas de los "costes ocultos» de las dinámicas migratorias.

Las «familias transnacionales» exigen una reacomodación permanente de las relaciones de género y generacionales en cuanto a los vínculos económicos, afectivos y de poder dentro del grupo doméstico, tanto en los lugares de origen como de destino (Pedone, 2008). Tales articulaciones están fuertemente condicionadas por el género. Las mujeres desempeñan un papel clave en dichos procesos, por cuanto ejercen de pivotes del mantenimiento de los lazos familiares, a la vez que actúan de sostén económico de sus familiares. En definitiva, constituyen el «nodo» intergeneracional que permite mantener la cohesión del grupo familiar y las redes migratorias (Baby-Collins, Cortes y Sassone, 2008).

Tras analizar los arreglos y las formas de gestión de los vínculos a distancia de las familias transnacionales bolivianas, se ponen de manifiesto tanto las dificultades a las que se enfrentan a la hora de minimizar los riesgos y los impactos negativos, como el potencial transformador de los significados y las prácticas que se dan en su seno en cuanto a los roles de género. Todo ello se concreta de forma diferente en función de:

- Los parámetros de género que marcan el proceso migratorio, es decir, de si se trata de estructuras familiares en las que ha emigrado el varón, o bien si se trata de una mujer "pionera». Para el caso boliviano, la influencia del género es especialmente perceptible, por cuanto los recientes patrones migratorios feminizados de Bolivia hacia España — y hacia Europa— han alterado sustancialmente los cánones de la "cultura migratoria» de la sociedad boliviana.

- Las estrategias para asegurar la reproducción social y lograr los objetivos del grupo se basan en relaciones de intercambio y reciprocidad. Sus impactos difieren según de qué miembro de la familia se trate y, de acuerdo con Bernhard et al. (2005), de los recursos tanto materiales como inmateriales de los que se disponga, tanto en origen como en destino (recursos de clase, momento del ciclo vital, calidad de las redes familiares, etc.).

- Para el caso específico de las transformaciones en las relaciones de género desde la perspectiva de las mujeres, sus experiencias en los procesos 
migratorios pueden ser muy diferentes y oscilan desde el empoderamiento hasta la pérdida de estatus social (Magliano, 2007). Sin embargo, qué duda cabe que su participación en dichos procesos entraña en sí misma un potencial de cambio, tanto si ejercen de left-behind como si son ellas las que han emigrado. Para estas últimas, las mujeres "pioneras», a pesar de que su estructura de oportunidades en España se ve claramente afectada por patrones de subordinación laboral (todas las mujeres migrantes entrevistadas se emplean en el servicio doméstico) y de que su significado «emancipador» tiene que interpretarse a la luz de su estatus social premigratorio, los resultados presentados sí muestran la presencia de un discurso de "empoderamiento", argumentado desde su papel como principales sustentadoras del grupo familiar a consecuencia del proyecto migratorio.

Asimismo, a nivel metodológico, más allá de la conveniencia de utilizar la familia transnacional como unidad de análisis, hay que reflexionar sobre los límites del marco de estudio bipolar (Bolivia y España) utilizado en esta investigación para captar los vínculos transnacionales. En este sentido, se hace necesario adaptar los dispositivos metodológicos de la perspectiva transnacional, con el fin de poder captar los actuales patrones migratorios en toda su complejidad. Como se ha mostrado para el caso del contexto migratorio boliviano (Hinojosa, 2009b), estos patrones se concretan cada vez más desde dinámicas y marcos multipolares o multilocalizados, que sobrepasan los campos sociales definidos exclusivamente desde una sociedad de origen y una sociedad de destino.

\section{Referencias bibliográficas}

AliCEA, Marixsa (1999). "A Chambered Nautilus": The Contradictory Nature of Puerto Rican Women's Role in the Social Construction of a Transnational Community». Gender and Society, II (5), 597-626.

ARIZA, Marina (2000). Ya no soy la que dejé atrás...: Mujeres migrantes en República Dominicana. México: Plaza y Valdés.

Baby-Collins, Virginia; CorTes, Geneviève y Sassone, Susana (2008). «Mujer, movilidad y territorialización: Análisis cruzado de las migraciones internacionales en México y Bolivia». En: Godard, H. y SAndoval, G. (eds.). Migración transnacional de los Andes a Europa y Estados Unidos. Lima / La Paz: IFEA/PIEB/IRD, 135-166.

BALÁN, Jorge (1990). «La economía doméstica y las diferencias entre los sexos en las migraciones internacionales: Un estudio sobre el caso de los bolivianos en la Argentina». Estudios Migratorios Latinoamericanos, 15-16, 269-295.

BALDASSAR, Loretta (2008). «Missing Kin and Longing to be Together: Emotions and the Construction of Co-presence in Transnational Relationships». Journal of Intercultural Studies, 29 (3), 247-266.

Basch, Lina; Glick Shiller, Nina y Szanton Blanc, Cristina (1994). Nations unbound: Transnational projects, postcolonial predicaments and deterritorialized nation-states. Pensilvania: Gordon and Breach Science Publishers. 
Bastia, Tanja (2007). «From Mining to Garment Workshops: Bolivian Migrants in Buenos Aires». Journal of Ethnic and Migration Studies, 33 (4), 655-669.

Benería, Lourdes (2008). "The Crisis of Care, International Migration and Public Policy». Feminist Economics, 14 (3), 1-21.

BERNHARD, Judith et al. (2005). "Transnational, multi-local motherhood: Experiences of separation and reunification among Latin american families in Canada». CERIS WP, 40 [en línea]. <http://www.ryerson.ca/\%7Ebernhard/documents/ WorkingPaperSeries.pdf $>$.

Bryceson, Deborah y VuOrela, Ulla (eds.) (2002). The Transnational Family: New European Frontiers and Global Networks. Oxford: Berg.

CACHÓN, Lorenzo (2009). La "España inmigrante»: Marco discriminatorio, mercado de trabajo y politicas de integración. Barcelona: Anthropos.

CORTES, Geneviève (2004). Partir para quedarse: Supervivencia y cambio en las sociedades campesinas andinas de Bolivia. La Paz: IRD, Plural, IFEA.

DANDlER, Jorge y MedeIRos, Carmen (1991). «Migración temporaria de Cochabamba, Bolivia a la Argentina: Patrones e impacto en las áreas de envío». En: PESSAR, P. R. (ed.). Fronteras permeables: Migración laboral y movimientos de refugiados en América. Buenos Aires: Planeta.

Di LeOnARDO, Micaela (1992). "The female world of cards and holidays: Women, families, and the work of kinship». En: THORne, B. y Yalom, M. (eds.). Rethinking the family: Some feminist questions. Boston: Northeastern University Press.

Dreby, Joanna (2007). «Children and Power in Mexican Transnational Families». Journal of Marriage and Family, 69, 1050-1064.

FE Y ALEGRía (2010). Los efectos de la migración de los progenitores en el desempeño escolar de los niños y adolescentes en Bolivia. Madrid: Entreculturas.

Gadea, Elena; Benencia, R. y Quarante, G. (2009). «Bolivianos en Argentina y en España: De la migración tradicional a las nuevas rutas». AREAS. Revista Internacional de Ciencias Sociales, 28, 30-43.

Glick SCHILler, Nina; BASCH, Linda y SzANTON-BlanC, Cristine (1992). «Towards a Definition of Transnationalism: Introductory Remarks and Research Questions». En: Glick Schiller, N.; Basch, L. y Szanton-Blanc, C. (eds.). Towards a Transnational Perspective on Migration: Race, Class, Ethnicity and Nationalism Reconsidered. Nueva York: New York Academy of Sciences.

GONZÁlVEZ, Herminia (2005). "Familias y hogares transnacionales: una perspectiva de género". Puntos de Vista, 11, 7-26.

GrasmucK, Sherri y Pessar, Patricia (1991). Between Two Islands: Dominican International Migration. Berkeley: University of California Press.

Gregorio, Carmen (1998). La migración femenina y su impacto en las relaciones de género. Madrid: Narcea.

GuARnizO, Luis Eduardo (1997). "Going Home: Class, Gender and Household Transformation Among Dominican Return Migrants». En: Pessar, P.R. (ed.). Caribbean Circuits: New Directions in the Study of Caribbean Migration. Nueva York: Center for Migration Studies.

Harrington, Mona (1999). Care and Equality, Inventing a New Family Politic. Nueva York: Routledge.

Herrera, Gioconda (2004). «Elementos para una comprensión de las familias transnacionales desde la experiencia migratoria del Sur del Ecuador». En: Hidalgo, F. (ed.). Migraciones: Un juego con cartas marcadas. Quito: ILDISAbya Yala. 
Herrera, Gioconda (2005). «Mujeres ecuatorianas en las cadenas globales del cuidado». En: Herrera, Gioconda; Carrillo, Cristina y Torres, Alicia (eds.). Migración ecuatoriana: Redes, transnacionalismo e identidades. Quito: FLACSO-Plan Migración Comunicación y Desarrollo.

- (2008). «Mujeres ecuatorianas en el trabajo doméstico en España». En: NovicK, Susana (comp.). Las migraciones en América Latina. Buenos Aires: Consejo Latinoamericano de Ciencias Sociales (CLACSO).

Hinojosa, Alfonso (2008a). «Transnacionalismo y multipolaridad en los flujos migratorios de Bolivia: Familia, comunidad y nación en dinámicas globales». En: Godard, H. y Sandoval, G. (eds.). Migración transnacional de los Andes a Europa y Estados Unidos. Lima / La Paz: IFEA/PIEB/IRD, 77-101.

- (2008b). España en el itinerario de Bolivia: Migración transnacional, género y familia en Cochabamba. En: NovicK, Susana (comp.). Las migraciones en América Latina. Buenos Aires: Consejo Latinoamericano de Ciencias Sociales (CLACSO).

- (2009a). Buscando la vida: Familias bolivianas transnacionales en España. Estado de situación. La Paz: CLACSO / Fundación PIEB.

- (2009b). «Migración boliviana a España: antecedentes, caracterización y perspectivas». En: AA.VV. Migraciones contemporáneas: Contribución al debate. La Paz: CIDES-UMSA, 157-180.

Ho, Elsie (2002). «Multi-local residence, transnational networks, Chinese astronaut families in New Zealand». Asian and Pacific Migration Journal, 11 (1), 145-164.

HochsCHILD, Arlie Russell (1983). The Managed Heart: Commercialization of Human Feeling. Berkeley: University of California Press.

- (2003). The Second Shift. Nueva York: Penguin Group.

Hondagneu-Sotelo, Pierrete (1994). Gendered Transitions: Mexican Experiences of Migration. Berkeley y Los Ángeles: University of California Press.

- (2000). «La incorporación del género a la migración: no sólo para feministas ni sólo para la familia». En: BARrera, Dalia y OEHMiChen, Cristina (eds.). Migración y relaciones de género en México. México DF: Grupo Interdisciplinario sobre Mujer, Trabajo y Pobreza (GIMTRAP) / UNAM, Instituto de Investigaciones, 423-451.

INSTRAW (2005). Estudio sobre migración internacional y remesas en Colombia [en línea]. Bogotá: OIM-Colombia. <http://tukuymigra.com/images/normativa/ COL_EST_OIM-DANE_4.pdf>.

LANDOLT, Patricia (2006). «Modernidad tardía y migración transnacional: reflexiones conceptuales desde el caso de El Salvador». En: ArdiLA, G. (ed.). Colombia: Migraciones, transnacionalismo y desplazamiento. Bogotá: Universidad Nacional de Colombia.

LevitT, Peggy y Glick Schiller, Nina (2004). "Conceptualizing Simultaneity: a Transnational Social Field Perspective». International Migration Review, 38, 10021038.

Magliano, María José (2007). «Migración de mujeres bolivianas hacia Argentina: Cambios y continuidades en las relaciones de género". Amérique Latine Histoire et Mémoire. Les Cahiers ALHIM [en línea], 14, <http://alhim.revues.org/index2102. html>.

Magliano, María José y Romano, Silvina (2011). «El desarrollo y las migraciones femeninas en la agenda política sobre migraciones internacionales: Universalismo etnocéntrico y colonialidad de género». En: Pizarro, C. (coord.). Migraciones internacionales contemporáneas: Estudios para el debate. Buenos Aires: Mingione, Enzo. 
Moore, Henrietta L. (1999). Antropología y feminismo. Madrid: Cátedra.

Oso, Laura (1998). La migración hacia España de las mujeres jefas de hogar. Madrid: IMU.

— 2007). «Migración, género y hogares transnacionales». Ponencia presentada en el $4^{\circ}$ Congreso sobre la Inmigración en España. Valencia, 21-23 de marzo de 2007.

PARELLA, Sònia (2007). «Los vínculos afectivos y de cuidado en las familias transnacionales: Migrantes ecuatorianos y peruanos en España». Migraciones Internacionales, 4 (2), 39-76.

Parella, Sònia y CaVAlCANTI, Leonardo (2010). «Dinámicas familiares transnacionales y migración femenina». En: GRUPO INTERDISCIPLINARIO DE INVESTIGADOR@S MigRANTES (coord.). Familias, niños, niñas y jóvenes migrantes: Rompiendo estereotipos. Madrid: IEPALA.

PARREÑAS, Rachel Salazar (2000). «Migrant Filipina Domestic Workers and the International Division of Reproductive Labor». Gender \& Society, 14 (4), 507-524.

- (2001). Servants of Globalization: Women, Migration and Domestic Work. Palo Alto (CA): Standford University Press.

- (2003). "The Care Crisis in the Philippines: Children and Transnational Families in the New Global Economy». En: Ehrenreich, B. y Russell Hochschild, A. (eds.). Global Woman. Londres: Granta Books.

- (2005). Children of Global Migration: Transnational Families and Gendered Woes. Stanford, CA: Stanford University Press.

PajARES, Miguel (2010). Inmigración y mercado de trabajo. Informe 2010 Madrid: Observatorio Permanente de la Inmigración.

Pedone, Claudia (2004). «Negociaciones en torno al asentamiento definitivo de las familias migrantes ecuatorianas: Construcción de espacios sociales transnacionales». Ponencia presentada en el $4^{\circ}$ Congreso sobre la Inmigración en España. Girona, 10-13 de noviembre de 2004.

— (2008). "Varones aventureros” vs. "Madres que abandonan”: Reconstrucción de las relaciones familiares a partir de la migración ecuatoriana». REMHU. Revista Interdisciplinar da Movilidade Humana, 30, 45-64.

PEÑA, Lourdes et al. (2003). Interculturalidad entre chapacos, quechuas, aymaras y cambas en Tarija. La Paz: Fundación PIEB.

Pessar, Patricia R. y MAHLER, Sarah J. (2003). "Transnational migration: bringing gender». International Migration Review: IMR, 37 (3), 812-846.

PorTes, Alejandro; GuarnizO, Luis Eduardo y LANDOlT, Patricia (1999). «The Study of Transnationalism: Pitfalls and Promise of an Emergent Research Field». Ethnic and Racial Studies, 2, 217-237.

- (2003). La globalización desde abajo: Transnacionalismo inmigrante y desarrollo. México D.F.: FLACSO.

PRIBILSKY, Jason (2004). “Aprendamos a convivir”: conjugal relations, coparenting and family life among Ecuadorian transnational migrants in New York City and the Ecuadorian Andes». Global Networks, 4 (3), 313-334.

PuYAnA, Yolanda et al. (2009). Entre aqui y allá: Las familias colombianas transnacionales. Bogotá: Fundación Esperanza.

Quijano, Aníbal (2005). «Colonialidad del poder, eurocentrismo y América Latina». En: LANDER, E. (comp.). La colonialidad del saber: eurocentrismo y ciencias sociales. Buenos Aires: FLACSO, 201-246.

RiBAS, Natalia (2001). «Estrategias transnacionales?: Una pregunta acerca de las migraciones femeninas en España». Arxius de Ciències Socials, 1, 69-92. 
RoBerT, Elisabeth (2009). «Mujeres, migración, remesas y relaciones de género». En: AA.VV. Migraciones contemporáneas: Contribución al debate. La Paz: CIDESUMSA, 85-110.

RosAS, Carolina (2008). Varones al son de la migración: Migración internacional y masculinidades de Veracruz a Chicago. México: El Colegio de México.

- (2010). Implicaciones mutuas entre el género y la migración. Buenos Aires: EUDEBA/ Universidad de Buenos Aires.

SAsSEN, Saskia (1984). «The New Labor Demand in Global Cities». En: Smith, M.P. (ed.). Cities in Transformation. Beverly Hills: Sage, 139-171.

- (2003). Contrageografías de la globalización: Género y ciudadanía en los circuitos transfronterizos [en línea]. Madrid: Traficantes de Sueños. <http://www.edicionessimbioticas.info/IMG/pdf/contrageografias.pdf>.

SinATTI, Giulia (2008). «Migraciones, transnacionalismo y el locus de investigación: multi-localidad y la transición de "sitios" a "campos"». En: Solé, C.; PARELla, S. y Cavalcanti, L. (ed.). Nuevos retos del transnacionalismo en el estudio de las migraciones. Madrid: OPI.

SKRBIŠ, Zlatko (2008). «Transnational Families: Theorising Migration, Emotions and Belonging». Journal of Intercultural Studies, 29 (3), 231-246.

Solé, Carlota; PARElla, Sònia y CAVAlCANTI, Leonardo (2007). Los vínculos económicos y familiares transnacionales: Los inmigrantes ecuatorianos y peruanos en España. Madrid: Fundación BBVA.

SOLFrINI, Giuseppe (ed.) (2005). Tendencias y efectos de la emigración en el Ecuador, 3. Quito: ALISEI.

Sørensen, Ninna Nyberg (2007). «La vida de la familia transnacional a través del Atlántico: La experiencia de la población colombiana y dominicana migrante en Europa». Puntos de Vista, 9, 7-28.

Tienda, Marta y BoоTh, K. (2008). «Gender, Migration and Social Change». International Sociology, 6 (1), 51-72.

TOrre Ávila, Leonardo de la (2006). No llores, prenda, pronto volveré: Migración, movilidad social, herida familiar y desarrollo. La Paz: PIEB.

TORRE, Leonardo de la y Alfaro, Yolanda (2007). Caminos y sendas de desarrollo en los municipios migrantes de Arbieto y Toco. La Paz: PIEB.

VIDAL, Laura et al. (2002). «De Paraíso a Carolina del Norte: Redes de apoyo y percepciones de la migración a Estados Unidos de mujeres tabasqueñas despulpadoras de jaiba». Migraciones Internacionales, 1 (2), enero-junio.

WAGNER, Heike (2008). «Maternidad transnacional: discursos, estereotipos y prácticas». En: Herrera, G. y Ramírez, Jacques (ed.). América Latina Migrante: Estado, familia, identidades. Quito: Facultad Latinoamericana de Ciencias Sociales (FLACSO).

WHiteSELL, Lily (2008). "Y aquellos que se fueron: Retratos del éxodo boliviano». En: Schultz, J. y Crane Draper, M. (eds.). Desafiando la globalización: Historias de la experiencia boliviana. La Paz: El Centro para la Democracia / Plural Editores.

ZAREMBKA, Joy M. (2003). «America's Dirty Work: Migrant Maids and Modern-Day Slavery». En: EHRENREICH, B. y Hochschild, A.R. (ed.). Global Woman: Nannies, Maids, and Sex Workers in the New Economy. Nueva York: A Metropolitan and Own Book, 142-53.

Zimmerman, Mary K. et al. (2005). Global Dimensions of Gender and Carework. Palo Alto (CA): Stanford University Press.

ZONTINI, Elizabetta (2010). Transnational families, migration and gender: Moroccan and Filipino women in Bologna and Barcelona. Oxford: Berghahn Books. 